\title{
REPRESENTACIONES DE LA PROBLEMÁTICA AGRARIA EM LA PRENSA DE ANÁLISIS DE LOS AÑOS 70. PRIMEIRAS APROXIMACIONES COMPARATIVAS ENTRE EL DIARIO LA OPINIÓN (ARGENTINA) Y EL SEMANARIO OPINIÃO (BRASIL)
}

\author{
REPRESENTAÇÕES DA PROBLEMÁTICA AGRÁRIA NA IMPRENSA DE ANÁLISE \\ DOS ANOS 70. PRIMEIRAS APROXIMAÇÕES COMPARATIVAS ENTRE O DIÁRIO \\ LA OPINIÓN (ARGENTINA) E O SEMANÁRIO OPINIÃO (BRASIL)
}

AGRARIAN TROUBLES REPRESENTATIONS IN 70'S NEWSPAPERS. FIRST

COMPARATIVE APPROACHES BETWEEN LA OPINIÓN DAILY (ARGENTINA)

AND OPINIÃO (BRAZIL) WEEKLY

\begin{abstract}
Marina Poggi
Professor Associado Kennedy University
\end{abstract}

\section{Resumen}

La propuesta es analizar las representaciones discursivas elaboradas por el periódico $\mathrm{La}$ Opinión (Argentina) durante 1973 respecto de las reformas agrarias, y realizar una aproximación hacia el análisis de representaciones similares en el semanario Opinião (Brasil), para realizar un aporte al conocimiento de periódicos poco estudiados y sus roles como actores políticos.

Palabras clave: Prensa. Discurso. Reforma Agraria.

\section{Resumo}

A proposta é analisar as representações discursivas feitas sobre a reforma agrária pelo jornal La Opinión (Argentina) durante 1973 e das representações similares no semanário Opinião (Brasil) com vistas a contribuir numa temática pouco estudada, qual seja a de seus papéis como atores políticos.

Palavras-chave: Jornais. Discurso. Reforma Agrária.

\begin{abstract}
The proposal is to analyse the discursive representations made by the newspaper $L a$ Opinión (Argentina) during 1973 to agrarian reforms, and make an approach to the analysis of similar representations in the weekly Opinião (Brazil), to make a contribution to the knowledge of little-studied newspapers and their roles as political actors.
\end{abstract}


Keywords: Newspapers. Discourses. Agrarian Reform.

Esta obra está licenciada sob uma Licença Creative Commons

\section{INTRODUCCIÓN}

La importancia de estudiar medios de comunicación se funda en la presencia cotidiana que la prensa posee en la sociedad, una presencia que no se agota y se renueva cada día, ya que las noticias resultan de acontecimientos inmediatos: son elaboradas para el día y “no para durar en el tiempo” (Raiter, 2010: 21). Los medios, como uno de los “emisores institucionales por excelencia”, establecen la agenda; esto es posible por el carácter institucional de los enunciados que emiten y porque llegan a una gran cantidad de personas de modo simultáneo y con muy poco tiempo de diferencia (2010: 17). A la vez, los medios se convierten en uno de los focos de lucha ${ }^{1}$ en donde se disputa la hegemonía en torno a determinados significados, con el objetivo de ganar consentimientos e instalar significados específicos. Explorar minuciosamente tales enunciados y estimar las representaciones y los significados que construyen, resulta un aporte significativo para el conocimiento de la historia reciente.

El objetivo general de este trabajo es evaluar el rol de la prensa de análisis de la década de 1970 y su discurso acerca de la temática agraria. Esta prensa estaba inspirada principalmente en el estilo del semanario norteamericano Times y pensada para ser leída por minorías con intereses particulares, otorgándole un espacio prioritario al análisis de unos pocos temas antes que a brindar información variada de la actualidad.

En particular, la propuesta consiste en analizar las representaciones discursivas elaboradas por el periódico La Opinión respecto de las reformas agrarias -temática instalada en Latinoamérica desde 1960 y en Argentina fuertemente en 1970, particularmente en 1973 a partir de la restauración democrática del gobierno peronista-, y realizar una primera aproximación hacia el análisis de representaciones similares en el semanario Opinião de Brasil, con la intención de realizar un aporte al conocimiento de periódicos poco estudiados y sus roles como actores políticos. En cuanto a la problemática de la propiedad agraria, ni en Argentina ni en Brasil han ocurrido cambios

\footnotetext{
${ }^{1}$ Fairclough señala que la lucha hegemónica ocurre en un frente amplio, que incluye a las instituciones de la sociedad civil como las instituciones educativas, los sindicatos y la familia (1992: 92).
}

ANIMUS R. Interamericana de Comunicação Midiática, http://www.ufsm.br/revistas E-ISSN 2175-4977, v. 11, n. 21, Jan-Jun(2012) 
irreversibles de tipo "revolucionario" en sus sistemas de tenencia de la tierra. Tanto sus estructuras agrarias, lo mismo que sus reacciones ante los problemas agrarios, pueden considerarse, por lo tanto, representativos de la situación Latinoamericana del período estudiado (Barraclough y Domike, 1970: 120). Además, la política de tierras era revisada a nivel mundial desde la década de 1960, y la idea de una reforma agraria aparecía como una amenaza para los sectores latifundistas latinoamericanos, y por lo tanto cualquier política que intentara modificar el modo de tenencia de la tierra se volvía conflictiva y encontraba resistencias. Así, el estudio pretende constituirse en material empírico que dé cuenta no sólo de "que dice” la prensa en relación a una determinada temática, sino fundamentalmente en "como lo dice”.

La elección de los dos medios responde a que ambos poseen características similares, tanto en su estética copiada del diario francés Le Monde, como en contenidos y en el público lector al que se dirigen. Por otra parte, ambos semanarios nacen -con un año de diferencia- en contextos políticos conflictivos, y finalizan en el mismo año bajo los efectos de la censura y la clausura de las instalaciones por la acción de gobiernos autoritarios. Al igual que La Opinión, Opinião le daba prioridad al análisis y al debate intelectual antes que a la información. Con un formato idéntico al del periódico argentino, que incluía ilustraciones y caricaturas en vez de de fotografías, ambas publicaciones procuraban imprimir una diferencia en el tratamiento de la información respecto de la prensa tradicional que circulaba en ese período. La elaboración de sus discursos y la posible influencia de tales discursos en las decisiones políticas, es un aspecto que merece ser estudiado, contemplando que ambos periódicos marcaron el inicio de una nueva prensa crítica y de análisis, un formato que fue innovador para el estilo tradicional de la prensa gráfica de sus respectivos países.

Como herramientas metodológicas se utilizarán algunas categorías propuestas por el Análisis Crítico del Discurso. El ACD estudia el lenguaje como práctica social, es decir como una forma de significar a un particular ámbito de la práctica social desde una particular perspectiva, y considera que el contexto del uso del lenguaje es crucial. De este modo, las prácticas sociales configuran los discursos y los afectan, y los discursos influyen en las acciones y los procesos sociales y políticos (Wodak, 2003: 104). Para el ACD "las estructuras dominantes estabilizan las convenciones y las convierten en algo natural, es decir, los efectos del poder y de la ideología en la 
producción de sentido quedan oscurecidos y adquieren formas estables y naturales” (2003: 19-20).

Para estudiar el escenario de la prensa, se utilizará la metáfora de la "escena enunciativa” propuesta por Irene Fonte (2003) para referirse al despliegue de voces que conforman la dimensión política de un acontecimiento en la prensa. La autora plantea que "los políticos involucrados parecen interactuar en un escenario descrito por la prensa o imaginado por el lector” (2003: 66). De este modo, las voces se incorporan en la escena de modo directo o de modo indirecto. A la vez, el receptor de las noticias puede también formarse una representación mental de la escena juntando varias noticias sobre un mismo asunto, con diversos actores que opinan sobre la misma cuestión, al tiempo que observa como el tema se prolonga en el tiempo: así, la escena enunciativa va creciendo, aumenta la referencia a discursos previos y se forma una compleja red de citas.

\section{CONTEXTO HISTÓRICO}

En relación al contexto internacional, entre las décadas de 1960 y 1970, comenzaron a percibirse transformaciones en la economía del mundo capitalista que advertían el inicio de un período de crisis y empezaron a manifestarse problemas en el plano productivo, que fueron los causantes de la ruptura de la "edad de oro del capitalismo” (Rapoport, 2005: 505). La escena mundial estaba plagada de protestas desencadenadas por el "mayo" francés y las movilizaciones contra la Guerra de Vietnam en los EE.UU, y con el condimento de la amenaza del avance del comunismo debido al triunfo de la revolución cubana a principios de 1959. En Argentina, el gran estallido social tuvo lugar en Córdoba el 29 de mayo de 1969 y fue conocido como "el Cordobazo” (Rapoport, 2005: 445 y 514).

El inicio del nuevo período democrático en 1973 coincidió con la llegada del peronismo al poder, que cambió el régimen político del país de autoritario a democrático y el periodismo argentino se encontró “con un ámbito de libertad que no vivía en décadas”. Tal transición produjo un cambio en las formas públicas de circulación de la información política. La red de fuentes cambió “por el surgimiento del Congreso, el que se convirtió -al menos al principio del nuevo régimen- en caja de 
resonancia informativa de todos los acontecimientos políticos”, y por el repliegue de los actores militares y sus particulares formas de difundir la información (Ruíz, 2001: 133).

La propuesta preelectoral de 1973 buscaba dar una respuesta a la creciente “presión nacional sobre la tierra”, y proponía políticas tendientes a lograr un aumento en la producción y en la productividad agropecuaria, “a través de la puesta en producción de toda la tierra con aptitud para la explotación agropecuaria” (Lattuada, 1986: 214). De este modo, se reconocía como objetivo fundamental la necesidad de elevar los niveles de productividad y volúmenes globales de la producción agropecuaria, y el logro de este objetivo se encontraba asociado a la corrección de deficiencias estructurales permanentes en la estructura agraria, entre las cuales se destacaban e interrelacionaban el sistema de tenencia de la tierra y la subutilización de suelo como factor productivo (1986: 215).

Las políticas agrarias proyectadas que regularían el uso y la tenencia de la tierra en Argentina se inscriben dentro del paquete de medidas que fueron consideradas por los sectores terratenientes como reformistas, $\mathrm{y}$ en ese sentido se entendieron por tales sectores como un atentado al derecho constitucional de propiedad privada. La idea rectora de las propuestas era que la tierra debía ser para el que la trabajara; tal expresión significaba que la tierra debía ser un bien de producción y no un bien de renta y especulación.

Estos planteos, contenidos en la plataforma electoral de 1973, fueron denominados como "Reforma Agraria Integral”, y profundizados en las Pautas Programáticas para el Gobierno Justicialista de la Reconstrucción Nacional, texto en el que se ratificaba el principio de que la tierra debe ser para quien la trabaja, alentando al acceso de los productores a la propiedad y a la erradicación de los latifundios y los minifundios improductivos.

Al llegar el gobierno peronista, de las propuestas preelectorales se pasó al dictado de medidas concretas. En primer lugar, la Ley 20.518 -conocida como Ley de suspensión de Juicios de desalojos-, suspendía desde julio de 1973 y hasta diciembre de 1974 los trámites de juicios de desalojo de los arrendatarios rurales. La Ley 20.538 establecía que a partir del 1 de enero y hasta el 31 de diciembre de 1974 regiría un impuesto nacional de emergencia sobre las tierras libres de mejoras (ITLM) y, a partir del 1 de enero de 1975, regirían las modificaciones para determinar la renta neta de las 
explotaciones (IRNP). Luego, la ley 20.543 -denominada de "Fomento Agrario"concedía créditos a arrendatarios y aparceros para la compra de medios de trabajo. Todavía en el plano de las propuestas, el Anteproyecto de Ley Agraria trataba de reunir orgánicamente, en un solo instrumento jurídico, la acción del Estado para regular el dominio, el uso y la tenencia de la tierra.

Es posible agrupar para su análisis al conjunto de las políticas agrarias (tanto leyes como proyectos) dentro de este plan de Reforma Agraria, contemplando que estas medidas si bien apuntaban a maximizar la productividad de las tierras, de un modo u otro afectaban la estructura de la propiedad. Por este motivo, del corpus total se seleccionaron para el análisis en este capítulo las cuarenta y ocho noticias en las que aparece la noción de reforma agraria como conjunto de medidas reformistas.

El diario La Opinión se hizo eco de las medidas propuestas que regularían el uso y la tenencia de la tierra, respondiendo a los intereses y vinculaciones económicas que el dueño del diario tenía con el Ministro de Economía Gelbard (Mochkofsky, 2004; 159). Con el estilo periodístico norteamericano de Times e inspirado en la estética de Le Monde, La Opinión fue creado el 4 de mayo 1971 por el periodista Jacobo Timerman en un contexto político nacional conflictivo y cambiante, con la intención, tal como lo anuncia su lema, de ser "el diario independiente de la mañana”; la característica principal era la información con análisis, dándole un lugar importante al juicio de los periodistas. Era un proyecto periodístico que intentaba competir con otros medios en cuanto a cantidad de información, sino que prefería ocuparse de pocos temas, pero en profundidad y con una mirada universal: su objetivo principal era formar opinión y para ello no ofrecía noticias, sino análisis (Díaz, 2002: 143).

Además de fundarlo, Timerman fue quién dirigió la publicación, y contó entre sus filas con Hermenegildo Sabát en las ilustraciones y caricaturas, Horacio Verbitsky como jefe de redacción, Juan Carlos Algarañáz en política nacional y Jorge Riaboi, Arcadio Oña y Luis Felipe Sapag en economía. Entre otras figuras destacadas, participaron en sus páginas Juan Gelman y Miguel Bonasso, quién ingresó para cubrir de forma exclusiva la llegada de Héctor Cámpora al poder (Mochkofsky, 2004; 185). En su mayoría, los integrantes de La Opinión coincidían ideológicamente con la militancia peronista o de izquierda. Por ejemplo, Verbitsky, Sapag y Bonasso militaron en la organización guerrillera peronista Montoneros, Algañaráz simpatizaban con la izquierda 
peronista y Gelman se unió a la FAR (Fuerzas Armadas Revolucionarias). El proyecto de La Opinión llegó a su fin el 25 de mayo de 1977, cuando su director y fundador Timerman fue secuestrado, y el diario clausurado y expropiado por el gobierno militar.

Por su parte, el semanario Opinião apareció en Brasil en noviembre de 1972 y permaneció hasta abril de 1977. A diferencia de La Opinión, el semanario brasilero nace en un período en el que el país se encontraba bajo el mando del poder militar desde 1964, y en 1973 la presidencia estaba ocupado por HYPERLINK "http://es.wikipedia.org/wiki/Emilio_Garrastazu_M\%C3\%A9dici" lo "Emilio Garrastazu Médici" Emilio Garrastazu Médici de la Aliança Renovadora Nacional, partido político creado para dar sustento a tal poder militar (Schilling, 2009).

En cuanto al panorama agrario de Brasil, hacia 1970 enfrentaba un proceso modernizante que contemplaba diversas políticas agrícolas iniciadas por Delffim Netto Ministro de Hacienda en 1973 y Ministro de Agricultura pocos años después- que inducían al crecimiento de la producción agrícola en una industria de insumos y máquinas volcados hacia la agricultura, pero con un crecimiento funcional a la industrialización. Las principales características negativas de ese proceso fueron la desregulación del uso del territorio y el carácter predatorio en relación a los recursos naturales y las fronteras agrícolas, imponiendo un elevado costo social y ambiental a la sociedad (Goulat Árabe, 2008: 35).

Opinião, que surge entonces como espacio contestatario hacia el gobierno militar, era editado por Fernando Gasparian, un intelectual y defensor de la democracia y, entre los colaboradores más conocidos, contó con el sociólogo Fernando Henrique Cardoso $^{2}$ (quién luego fue presidente), el escritor mexicano Octavio Paz, el sociólogo argentino Torcuato di Tella y el sociólogo francés Alain Touraine ${ }^{3}$. El semanario intentaba “ampliar los límites de las libertades públicas”, y en La Opinión fue presentado como "un semanario que aparece como edición brasileña del periódico francés Le Monde” (Ruiz, 2001; 48) y se publicitaba antes de lanzar su primer número como "Um jornal que não defende interesses pessoais, não pertence a nenhum partido, não é porta-voz de qualquer ideologia e se recusa a aceitar um volume de publicidade que ultrapasse a 20 por cento de sua receita. Esses são os princípios básicos de

\footnotetext{
${ }^{2}$ http://www.santistaroxo.com.br/artigo/?id=123. Consulta: 27/03/2011

3 http://www.independent.co.uk/news/obituaries/fernando-gasparian-421600.html.Consulta: $27 / 03 / 2011$
}

ANIMUS R. Interamericana de Comunicação Midiática, http://www.ufsm.br/revistas E-ISSN 2175-4977, v. 11, n. 21, Jan-Jun(2012) 
OPINIÃO”( Marquardt, 1999). En este sentido, la publicación le dedicó un espacio importante a la temática de las reformas agrarias a nivel mundial, a la problemática de la tierra, como así también dedicó una amplia cobertura al proceso de cambio político argentino iniciado con el regreso del peronismo al poder.

\section{LA ESCENA ENUNCIATIVA DE LA REFORMA AGRARIA}

Considerando el contexto argentino señalado en el inicio de 1973, y en función de que la problemática de la modalidad de tenencia de la tierra era un tema vigente en el marco de los debates preelectorales, se distinguen en La Opinión un total de 13 noticias que contienen la expresión reforma agraria. En cambio en Opinião, esta referencia no aparece de modo tan preciso dado que en el contexto político brasilero la reforma agraria no se debatía como un tema central: por este motivo, se consideraron para el análisis 14 noticias en las que sí se registran referencias a la reforma agraria, pero también pueden identificarse expresiones relacionadas con la propiedad de la tierra en diversas noticias que abarcan temas generales, expresiones relacionadas a los "sin tierra", y en los casos en los que cubre la situación argentina se refiere a las medidas reformistas que aplicaría el gobierno peronista.

\subsection{REPRESENTACIONES DE LA REFORMA AGRARIA EN LA OPINIÓN}

En el diario La Opinión es posible diferenciar voces propias y ajenas, y la intencionalidad de incorporar una u otra de las voces opera en función de construir un efecto de sentido determinado. Esta diferenciación de voces se encuentra marcada en el contraste de las notas que llevan la firma del periodista -donde se incorporan citas textuales y enunciados referidos-, y las que no lo hacen -donde queda reflejado el propio discurso del diario ${ }^{4}$.

Durante el año estudiado La Opinión publica trece noticias que contienen la expresión reforma agraria, y siete las que aparecen sin firma durante el período estudiado. Entre estas últimas se registran diversas formas para referise a la posibilidad de implementar una reforma agraria: debate, estudio, impuesto a la tierra, búsqueda de una reforma pacífica y ordenada, eliminación de latifundios, criterios superadores en cuanto a productividad y minifundios.

\footnotetext{
${ }^{4}$ En el caso estudiado es posible definir la postura que el periódico asume en relación a una posible reforma agraria, entendiendo que las noticias que no poseen firma, y que tampoco incorporan un discurso ajeno en -los términos en que Voloshinov lo propone-, o que no añaden intertextualidad manifiesta -en términos de Fairclough (1992:117)- serían las que pueden acercarse a definir la postura ideológica de La Opinión en cuanto a la reforma agraria, operando como la línea editorial del periódico (o como un espacio editorial diseminado por todo el diario, espacio que habitualmente aparece concentrado en los diarios solo en la línea editorial).
}

ANIMUS R. Interamericana de Comunicação Midiática, http://www.ufsm.br/revistas E-ISSN 2175-4977, v. 11, n. 21, Jan-Jun(2012) 
Por ejemplo, en la nota publicada el 17 de mayo -titulada "Estudian 93 entidades el impuesto a la tierra” ${ }^{5}$ - el concepto de reforma agraria se vincula con los de debate y estudio, además de incluir en el concepto de reforma -como una afirmación- al impuesto a la tierra. En el cuerpo de la noticia se argumenta que El impuesto a la tierra es componente fundamental de cualquier reforma agraria.

Luego, el $1^{\circ}$ de julio, y relacionado con el debate parlamentario en torno a las reformas económicas, se expresa que

\begin{abstract}
Más evidentes son las influencias ejercidas en contra de la ley que establece el impuesto a la renta normal potencial de la tierra. Algunos legisladores plantean una reforma agraria total mediante la expropiación de las tierras, cuando el proyecto consiste en realidad en una reforma agraria en sí mismo, pues obligará a los terratenientes, por el enorme peso de la carga impositiva que deberán afrontar, a deshacerse de las extensiones que no produzcan o conserven a título especulativo. Las entidades ruralistas se han puesto unánimemente en contra del proyecto, aún aquellas que por su extracción deberían acompañar esta medida de avanzada, y exigen que el impuesto a la renta potencial no sea tomado como anticipo de réditos, con lo cual disminuiría grandemente su progresividad y su efecto redistribuidor. ${ }^{6}$
\end{abstract}

La noticia apunta a establecer un punto "conciliador" de posturas extremas que intentan influir en la política agraria, tanto de parte de quienes se oponen rotundamente al IRNP (llamando la atención a las entidades ruralistas como la FAA, que por sus reclamos históricos debería apoyar esta ley) como a quienes plantean una reforma agraria total (señalando que el propio proyecto consiste en una reforma agraria en sí mismo), ya que la tendencia de La Opinión hasta aquí evaluada consiste en apoyar la política económica propuesta por el gobierno de Cámpora.

De las seis notas que poseen firma, tres se refieren específicamente a la región pampeana y las tres restantes incorporan, en primer lugar, la opinión de un experto francés que cuestiona la política agrícola en América Latina, y luego a una serie de dos notas realizadas por un especialista que explica la crisis del concepto de reforma agraria.

Es notable considerar que la serie de dos notas -que aparecen en el mes de noviembre, con características retóricas más de explicación que de opinión-, poseen una

\footnotetext{
${ }^{5}$ La Opinión, "Estudian 93 entidades el impuesto a la tierra”, 17 de mayo de 1973, p. 13

${ }^{6}$ La Opinión, "Desde la izquierda y la derecha se ejerce una acción de pinzas contra los proyectos”, $1^{\circ}$ de julio de 1973, p. 12 
función didáctica dirigida a los lectores respecto de la conveniencia e importancia de repartir con criterio la renta de los impuestos aplicados y de realizar cambios respecto de la tenencia de la tierra. Cabe recordar que el 11 de septiembre se había sancionado la ley de Impuesto a la Renta Normal Potencial de la Tierra (20.538) y que tendría vigencia a partir del año siguiente.

Por último, la construcción de los predicados puede dividirse de acuerdo a cuatro criterios que dependen del enunciador: enunciadas por un experto, enunciadas por un periodista económico, enunciadas por el diario (notas sin firma) y citas y/o textos referenciales (notas con o sin firma).

Las predicaciones que aparecen directamente relacionadas con una reforma agraria en las tres notas en donde prima la voz de un experto son: 1) dudas sobre la efectividad de una reforma basada en el reparto igualitario de tierras; 2) cuestionamiento a las formas clásicas de reforma; 3) reforma del régimen impositivo agrario; 4) apoyo de la burguesía urbana al proceso de reforma, en busca del debilitamiento de terratenientes y de condiciones económicas favorables; 5) proceso.

Las vinculaciones directas a la expresión reforma agraria que se extraen de las citas tomadas por el diario son:

reconstrucción nacional (cita a ENA - Encuentro Nacional de Argentinos); eliminación del dominio latifundista (cita a ENA - Encuentro Nacional de Argentinos); estatización de los sectores claves de la economía (cita a ENA);

política exterior conveniente (cita a ENA);

desarrollo con justicia (cita al presidente Cámpora);

leyes de tierras ociosas (cita al presidente Cámpora);

colonización (cita al presidente Cámpora);

uso social y productivo de la tierra (cita al presidente Cámpora);

reforma a la ley de arrendamientos y suspensión de desalojos rurales (cita al presidente Cámpora).

Así, los fundamentos expresados por la dirigencia política fueron apoyados por La Opinión y operaron del siguiente modo: 1) Se presentaron los argumentos expuestos por la dirigencia política; 2) Los argumentos considerados como editoriales (aparecían 
en notas sin firma) coincidían con los dichos del gobierno; 3) Se publicaron notas firmadas por expertos en la temática agraria que coincidían y reforzaban los argumentos desde esta postura de especialista; 4) Se citaron argumentos de posturas ideológicas de izquierda, que además coincidían con la postura y la propuesta oficial; 5) Los argumentos disconformes con la política oficial tuvieron una aparición limitada respecto a la temática abordada y aparecieron atenuados.

\subsection{REPRESENTACIONES DE LA REFORMA AGRARIA EM A OPINIÃO}

En Opinião las noticias relacionadas con una reforma agraria se pueden separar en tres grupos: las que mencionan el panorama agrario nacional, las que mencionan al panorama agrario mundial y las que mencionan la política agraria argentina.

En relación al primer grupo, las primeras noticia aparecen desde inicios de febrero bajo el interrogante de si finalmente se había iniciado una reforma, en correspondencia con la política de tierras planteado por el INCRA (Instituto Nacional de Colonización y Reforma Agraria) y la ejecución de PROTERRA. En una misma página, el semanario publica tres notas: en la primera de ellas plantea el interrogante mencionado mediante el recurso de inserción recurrente de discursos citados, en donde funcionarios del INCRA mencionan la propuesta como una política sabia con comprensión de empresarios y propietarios, pero la nota termina predicando que el futuro de la reforma es un misterio. ${ }^{7}$

La segunda nota contextualiza la problemática como un tema discutido por todos los gobiernos de la revolución ${ }^{8}$. En la última, aporta la opinión de un experto, Pompeu Accioly Borges, quién actúa como enunciador en la escena y detalla las características fundamentales que debe contener una reforma ${ }^{9}$, entre las que se destacan que la reforma agraria debe: ser un proceso masivo, rápido y drástico de redistribución de derechos sobre las tierras; movilizar las fuerzas políticas y entidades representativas de la población rural; adoptar un sistema económico de inversión; ser parte integral de un plan de desarrollo económico ${ }^{10}$.

Luego, en la última edición de julio, aparece una noticia en relación a la propuesta del INCRA para atraer a la región amazónica a la iniciativa privada. En

\footnotetext{
${ }^{7}$ Opinião, "Finalmente a reforma?", 5 a 12 de febrero de 1973, p. 7

${ }^{8}$ Opinião, “IBRA, INDA, INCRA, PROTERRA”, 5 a 12 de febrero de 1973, p. 7

9 Opinião, "Como evitar a reforma”, 5 a 12 de febrero de 1973, p. 7

10 Opinião, “Como evitar a reforma”, 5 a 12 de febrero de 1973, p.7
} 
relación a ello, la postura del semanario es crítica, y la estrategia que utiliza es la intensificación de los argumentos negativos, ubicados en la conclusión de la nota:

E frecuentemente uma carta denuncia detalhes insuspeitados, como a que Antonio Apolonio de Moura enviou, há dois meses, a seu compadre Mazinho, o deputado federal José Vieira Filho (ARENA$C E)$ : "Compadre: eu mando lhe dizer que aqui não se vê nada do que é prometido como eles dizem por aí". ${ }^{11}$

La siguiente noticia aparece en septiembre, y su tema central gira en torno a la posible expulsión de ocupantes de tierras del municipio de São Domingos de Capim, reclamadas por Pedro Alves dos Santos ${ }^{12}$. En este mismo sentido, hacia fin de mes otra nota refleja la resistencia armada en Maranhão ante el posible desalojo de tierras, y explica la problemática de los minifundios en la región ${ }^{13}$.

Por último, en el marco de noticias que abordan la cuestión de la agricultura brasilera, también es posible encontrar referencias a la modalidad de tenencia de la tierra, y observa que sería necessária

a reformulação da política agrícola do país, adequando-a ao desenvolvimento da economía no seu todo, sem o que graves problemas serão gerados pelas desigualdades de renda interregionais $^{14}$.

En esta dirección, en la misma edición y en la página siguiente de la noticia recién mencionada Chico de Oliveira realiza un análisis de la división y de la concentración de la tierra en Brasil, en la que el autor cuestiona la política oficial que, a partir de esquemas teóricos de la economía neoclásica, consideran a la agricultura como un espacio donde los factores económicos son libres y móviles, pero asume que el crecimiento, aunque lento, es siempre constante, y que el tiempo determinará el momento de correr. ${ }^{15}$

En el segundo grupo mencionado -las noticias que giran en torno a la problemática de la tierra a nivel mundial-, se registran tres noticias. La primera se publica en abril, está firmada por Charles Vanheche, y analiza el fracaso de la reforma Colombiana, frenada por el propio gobierno entre enero de 1970 y marzo de 1972,

${ }^{11}$ Opinião, "Incentivando a ocupção da Amazônia”, 30 del julio a 7 de agosto, p. 6

12 Opinião, "As terras em disputa”, 3 a 10 de septiembre , p. 4

13 Opinião, "As terras disputadas", 24 de septiembre a $1^{\circ}$ de octubre, p. 4

${ }^{14}$ Opinião, "Um setor em expectativa”, 10 de diciembre, p. 6

${ }^{15}$ Opinião, "O que é a agricultura brasileira?, 10 de diciembre, p. 7 
reforma que había sido lanzada en 1961, y hasta 1972 solo se habían expropiado 700 hectáreas. Si bien la noticia aparece firmada, es decir, con un periodista en el rol de enunciador, la estructura es ganada por citas textuales de diferentes actores colombianos que habían desarrollado el proyecto ${ }^{16}$. La segunda nota analiza la industrialización del campo en Bulgaria, dividida a partir de 1970 en 172 unidades agroindustriales gigantes -enfatizando en la especialización, la mecanización y la centralización- cuya oposición a la reforma agrícola se localizó en los jefes provinciales del partido comunista, quiénes sostenían que su poder sería diluido ante la presencia de técnicos, ingenieros y administradores agrícolas subordinados a los ministerios centrales. La noticia concluye expresando a la vez el escepticismo y la admiración hacia el funcionamiento de unidades agrícolas tan grandes, pero sostienen que el costo de ese tipo de agricultura sería muy alto, en función de la cantidad de personas necesarias para mantener su buen funcionamiento $^{17}$. Por último, en este grupo es posible ubicar una conmemoración realizada en diciembre por Opinião en los 25 del éxodo palestino, ya que en la nota resalta su situación, desde entonces, de "sin tierra"18.

En el tercer lugar, en el grupo caracterizado por las noticias que mencionan la política agraria argentina, la primera nota se registra en marzo, donde el semanario realiza una referencia a la política argentina en debate en términos de reformista y como consecuencia de la intención de reinstalar un gobierno populista ${ }^{19}$. En este mismo sentido, en el mes de julio, en una nota que analiza los cincuenta días de Héctor Cámpora en el gobierno, reproduce el reclamo de grupos revolucionarios opositores al gobierno (FAR - ERP), quienes consideraban que las reformas también deberían afectar los intereses de los monopolios y las oligarquías. ${ }^{20}$ Por último, en una nota publicada en noviembre, se menciona nuevamente la tipología de políticas reformistas adoptadas por el gobierno peronista, acentuando que la oligarquía rural se vería perjudicado por un aumento del $75 \%$ en el impuesto a la Renta Normal Potencial de la Tierra, el cual entraría en vigencia recién en 1975.

Entonces, luego de este recorrido por las noticias publicadas en Opinião que mencionan la problemática agraria, se puede concluir que:

\footnotetext{
${ }^{16}$ Opinião, “Côlombia: uma reforma agrária sem êxito”, 9 a 14 de abril, p. 13

17 Opinião, "A industrialização do campo", 30 de julio a 7 de agosto, p. 17

18 Opinião, "25 anos sem terra”, 21 de diciembre, p. 13

19 Opinião, "O que há de novo no peronismo?, 19 a 26 de marzo, p. 11

20 Opinião, “Os cinquenta días de Hector Campora”, 30 de julio a 7 de agosto, p. 16
} 
En el primer grupo, identificado como el de las noticias que mencionan la problemática de la tierra en Brasil, la intencionalidad se encuentra orientada a cuestionar la efectividad de la política de tierras ya implementada en el país.

En el segundo grupo, donde la información está centrada en el panorama mundial de la problemática de tierras, el análisis está centrado en momentos históricos ya pasados.

Las noticias que integran el último grupo, que contemplan las políticas peronistas, pueden se clasificadas como crónicas informativas, y las breves referencias a las políticas de tierras argentinas no poseen estrategias enunciativas específicas que busquen crear un efecto de sentido respecto de una posible reforma agraria.

\section{REFLEXIONES FINALES}

Si bien ambas publicaciones surgieron con muy poco tiempo de diferencia y mirando a un estilo de prensa nortemericana, pero con una estética copiada de Le Monde y la intención de dirigirse a un destinatario similar, las características contextuales particulares de cada uno de los países en que La Opinión y Opinião nacen, hacen que las dos publicaciones estudiadas tengan un rol político diferenciado. Así, mientras que La Opinión se inicia en un período argentino democrático, con la intención de conformar un espacio analítico de las noticias diarias, Opinião nace con la intención de convertirse en un espacio de análisis, pero contestatario hacia un gobierno militar que comienza a perder su fuerza.

El diario La Opinión encarnó para la prensa argentina un nuevo modelo de periodismo que resulta emblemático hasta la actualidad. El carácter analítico y las notas firmadas daban cuenta en su inicio de un período de libertad del que gozó el espacio público a principios de la década de 1970, libertad que se extinguió rápidamente en la mitad de la década. Aunque el estilo periodístico del diario no estuvo pensado para proclamarse a favor o en contra de temas agrarios, sino que fue pensado como medio de “formación” de opiniones para una "inmensa minoría”, se pudo observar en el estudio que el diario sí tomó una posición respecto de los temas agrarios, y esta postura llegó de la mano de la defensa de intereses económicos, asociados a las vinculaciones que el director de la publicación deseaba mantener con el poder. Así, La Opinión incorporó a 
la escena la multiplicidad de voces que conformaron el debate por la reforma agraria, pero se concentró en intensificar los argumentos propuestos por el peronismo gobernante.

Jacobo Timerman, dueño y fundador de La Opinión, le dio preferencia a las cuestiones empresariales del diario por sobre las ideológicas y procuró mantenerse cercano al poder de turno, y las estrechas relaciones con el Ministro de Economía José Ber Gelbard dejaron su huella en el diario en lo relacionado con el tratamiento de las noticias referidas a la temática agraria. Los argumentos esbozados por el diario para presentar y defender el Impuesto a la Renta Normal Potencial de la Tierra poseen mayores coincidencias argumentativas con la información publicada referente a las propuestas del presidente Héctor Cámpora en el mismo diario, y de este modo queda en evidencia una postura de apoyo a la política económica oficial.

En cuanto a Opinião, la actividad llevada a cabo durante 1973 fue acorde a la definida por su origen contestatario, de modo que en relación a las políticas agrarias, y a diferencia de La Opinión, la postura asumida no fue condescendiente con el gobierno y, muy sutilmente, utilizó el espacio para construir argumentos que inferían cuestionamientos a la política de tierras brasilera. Así, la problemática de la propiedad agraria estuvo presente en las páginas del semanario, aunque esta temática apareció diversificada: el problema de la tierra local por un lado, y las reformas agrarias mundiales por otro. A la vez, el semanario representó de manera profunda y sistemática el convulsionado momento de la política argentina, que incluyó tres cambios presidenciales y la cobertura de las propuestas preelectorales (que incluían la política de tierras) y el debate suscitado.

Por último, cabe mencionar que las estrategias argumentativas de La Opinión que procuraban apoyar a la política del gobierno de Cámpora, sostenidas durante el gobierno de Juan Domingo Perón, cambiarán sustancialmente luego del fallecimiento del líder justicialista, específicamente a partir del cambio del gabinete económico realizado por María Estela Martínez de Perón en su función como presidente. De este modo, mientras Opinião profundiza en las fisuras de un gobierno militar para abrirle camino a la democracia, a lo largo de su breve existencia La Opinión realiza cambios significativos en sus estructuras argumentativas, propiciando de algún modo desde sus páginas el pasaje de la democracia hacia la dictadura militar. 


\section{REFERÊNCIAS BIBLIOGRÁFICAS}

ANGENOT, M. El discurso social. Los límites históricos de lo pensable y lo decible. Buenos Aires: Siglo XXI, 2010.

BARRACLOUGH, S; DOMIKE A. La estructura agraria en siete países de América Latina, Reformas agrarias en América Latina. Buenos Aires: Juárez Editor, 1970. BEZERRA, A; CALLOU A. Estratégias de Comunicação nos programas de combate à pobreza rural no Nordeste, Intercom - Revista Brasileira de Ciências da Comunicação. São Paulo, v. 33, n.2, p.165-184, jul./dez.2009.

DÍAZ, C. La cuenta regressiva. Buenos Aires: La Crujía, 2002.

FAIRCLOUGH, N. Discourse and Social Change. Cambridge: Polity Press, 1992.

FONTE, I.La escena enunciativa en la prensa In: BERARDI, L. Análisis Crítico del Discurso. Perspectivas Latinoamericanas. México: Frasis Editores, 2003.

GOULAT ÁRABE, C. A inseraçao do tema agrario nas estratégias de desenvolvimento. Brasilia: MDA, 2008.

LATTUADA, M. La política agraria peronista (1943-1983). Tomos I y II, CEAL.

MARQUARDT, E. Opinião 1972-1973. Os limites regrados da oposição. In: Boletim de Pesquisa. Páginas do periodismo, v. 3, n. 4 - Florianópolis: NELIC, 1999.

MOCHKOFSKY, G. Timerman. Buenos Aires: Debolsillo (Sudamericana), 2004.

RAITER, A. Representaciones sociales. Versión corregida en enero de 2010 del trabajo original incluido en Representaciones Sociales (2001). Buenos Aires: EUDEBA, 2010.

RAPOPORT, M. Historia económica, política y social de la Argentina (18802003). Buenos Aires: Ariel, 2005.

RUIZ, F. Las palabras son acciones. Historia política y profesional de La Opinión de Jacobo Timerman (1971-1977). Buenos Aires: Perfil Libros, 2001.

SCHILLING, V. O bipartidarismo no regime militar. Disponível em: http://educaterra.terra.com.br/voltaire/brasil/2003/08/18/001.htm Acesso em: 17 de junho de 2011.

VERÓN, E. Construir el acontecimiento. Barcelona: Gedisa, 1987. 
WODAK, R. El enfoque histórico del discurso. In: WODAK, R. Métodos del Análisis Crítico del Discurso. Barcelona: Gedisa, 2003.

Original recebido em: 10/02/2011

Aceito para publicação em: 10/07/2012

\section{Resumo sobre o autor}

Doctora en Ciencias Sociales y Humanas por la Universidad Nacional de Quilmes (UNQ), Magíster en Ciencias Sociales y Humanidades con orientación en Comunicación, Especialista en Ciencias Sociales y Humanidades con Orientación en Comunicación Social y Licenciada en Comunicación Social. Profesora Adjunta de la Universidad Kennedy, Profesora Auxiliar de la Universidad Nacional de Quilmes e Investigadora del Centro de Estudios de la Argentina Rural (CEAR/UNQ). Buenos Aires, Argentina. 